\title{
Conclusions and General Summary
}

The toxicity of MAO inhibitors, particularly $<$ Tersavid $>$, as revealed in animal experiments is discussed and an attempt is made to relate the biochemical and pharmacological effects of this group of substances to their clinical action in angina pectoris. Various hypotheses concerning the action mechanism are advanced:

Rise in the level of 5-hydroxytryptamine in the blood with reduction of coronary resistance and augmentation of coronary flow (without increased oxygen consumption in the left ventricle); Increase in the storage capacity of the heart for catecholamines, possibly combined with suppression of the ischemic effect of these amines;

Reduction in the oxygen requirements of the myocardium (alterations in cardiac metabolism) or more economical use of oxygen;

Blocking of neurohumoral transmission (reduced pain conduction);

Possible participation of diamine-oxidase inhibition.

Of 594 evaluated cases of angina pectoris treated with Tersa-vid, 384 , i. e. $65 \%$ or approximately two-thirds responded successfully to the drug (definite reduction in the frequency and intensity of attacks).

The positive result is confirmed by a study carried out on the double-blind principle. The percentage of successful results would in all probability have been different if the choice of cases had not been decidedly negative in some of the groups reported and if lack of experience had not sometimes led to inadequate dosage of the drug.

In a number of cases with pathological ECG marked improvement in the ischemic signs appeared during treatment with Tersavid; in others the usual increase in such manifestations did not occur. These findings should be regarded cautiously, however, because of the relatively small number of cases and the complex nature of the problem.

Several patients with recent myocardial infarction were given Tersavid without adverse effect. The influence on residual pain was good in some cases, moderate in others.

Conclusions and General Summary

281

Tersavid does not interfere with anticoagulant therapy and patients requiring these drugs should continue to take them during treatment. Tersavid is also compatible with the therapeutic measures usual in angina pectoris. Apparently, Tersavid therapy should be given continuously, though in a number of cases the effect persisted for some time after medication was withdrawn. According to present experience, the drug retains its effect undimi-nished when administered for prolonged periods. One author mentions a favourable action in intermittent claudication.

The effective initial dose of Tersavid appears to be between 150 and $300 \mathrm{mg}$. a day, the daily maintenance dose about $100 \mathrm{mg}$. (50-200 mg. depending on the patient). The drug generally starts to take effect within the first week of treatment, but sometimes not until later. Tersavid is excellently tolerated. Side effects are insignificant and of the kind which may occur with any generally well tolerated tablet medication. Above all the drug has no notable hypotensive effect. Isolated hypotensive manifestations occurring in patients with recent 
myocardial infarction may freely be ascribed to the incident itself. Tersavid also shows only slight psychostimulation, so that it fulfils the two major requirements mentioned in the introduction.

Liver function tests, blood counts and urinalyses remained unremarkable during Tersavid therapy. Two patients with cirrhosis of the liver showed perfect tolerance of the drug. In one patient who contracted hepatitis during a hospital epidemic Tersavid therapy was not discontinued, and the hepatitis subsided without complications. One author carried out chemical investigations of the blood (determinations of lactic acid, pyruvic acid, adenosine triphosphoric acid, lactic acid dehydrogenase, SGO 1 and SGP 2 aminopherase); these were interesting in relation to a hypothetical mechanism of action proposed by the pharmacologists, but from the few provisional views put forward no definitive conclusions can be drawn.

In the same milligram for milligram dosage Tersavid is less effective than the prototype $<$ Marsilid > , but being so outstandingly well tolerated, it has a far wider therapeutic margin and can be given in correspondingly larger amounts. Since there is much in-

1 Serum glutamic acid oxalacetic acid.

2 Serum glutamic acid pyruvate.

282

Conclusions and General Summary

dividual variation in the response to this, as to other, forms of therapy, Tersavid covers a broad range of dosage requirements. This is a necessary prerequisite for the treatment of the ambulatory patient.

Because its effect is good, though not quite equal to that of the prototype, and because it is extremely well tolerated, Tersavid can be recommended as initial medication in the management of angina pectoris. If despite sufficiently prolonged treatment with sufficiently high doses of the drug no therapeutic effect is obtained, another MAO inhibitor, e.g. the prototype Marsilid, can be substituted, particularly where hypertension or depression of mood are associated symptoms. For those familiar with this form of therapy it is suggested that in cases of this kind Marsilid should be given in the first instance, in accordance with the recommendations in the supplement to Cardiologia vol. 35 . 